\title{
Strong anharmonicity in the phonon spectra of PbTe and SnTe from first principles
}

\author{
Guilherme A. S. Ribeiro, ${ }^{1,2}$ Lorenzo Paulatto, ${ }^{1}$ Raffaello Bianco, ${ }^{3,4}$ Ion Errea, ${ }^{5,6}$ Francesco Mauri, ${ }^{3,4}$ and Matteo Calandra ${ }^{1}$ \\ ${ }^{1}$ Institut de minéralogie, de physique des matériaux et de cosmochimie (IMPMC), Université Pierre et Marie Curie (Paris VI), \\ CNRS UMR 7590, IRD UMR 206, Case 115, 4 place Jussieu, 75252 Paris Cedex 05, France \\ ${ }^{2}$ The Capes Foundation, Ministry of Education of Brazil. Cx. postal 250, Braslia DF 70.040-020, Brazil \\ ${ }^{3}$ Dipartimento di Fisica, Università di Roma La Sapienza, Piazzale Aldo Moro 5, I-00185 Roma, Italy \\ ${ }^{4}$ Graphene Labs, Fondazione Istituto Italiano di Tecnologia, Via Morego, I-16163 Genova, Italy \\ ${ }^{5}$ Fisika Aplikatua 1 Saila, Bilboko Ingeniaritza Eskola, University of the Basque Country (UPV/EHU), \\ Rafael Moreno "Pitxitxi” Pasealekua 3, 48013 Bilbao, Basque Country, Spain \\ ${ }^{6}$ Donostia International Physics Center (DIPC), Manuel de Lardizabal pasealekua 4, 20018 Donostia-San Sebastián, Basque Country, Spain
}

(Received 10 September 2017; revised manuscript received 11 December 2017; published 10 January 2018)

\begin{abstract}
At room temperature, $\mathrm{PbTe}$ and $\mathrm{SnTe}$ are efficient thermoelectrics with a cubic structure. At low temperature, SnTe undergoes a ferroelectric transition with a critical temperature strongly dependent on the hole concentration, while $\mathrm{PbTe}$ is an incipient ferroelectric. By using the stochastic self-consistent harmonic approximation, we investigate the anharmonic phonon spectra and the occurrence of a ferroelectric transition in both systems. We find that vibrational spectra strongly depend on the approximation used for the exchange-correlation kernel in density-functional theory. If gradient corrections and the theoretical volume are employed, then the calculation of the phonon frequencies as obtained from the diagonalization of the free-energy Hessian leads to phonon spectra in good agreement with experimental data for both systems. In PbTe we evaluate the linear thermal expansion coefficient $\gamma=2.3 \times 10^{-5} \mathrm{~K}^{-1}$, finding it to be in good agreement with experimental value of $\gamma=$ $2.04 \times 10^{-5} \mathrm{~K}^{-1}$. Furthermore, we study the phonon spectrum and we do reproduce the transverse optical mode phonon satellite detected in inelastic neutron scattering and the crossing between the transverse optical and the longitudinal acoustic modes along the $\Gamma \mathrm{X}$ direction. The phonon satellite becomes broader at high temperatures but its energy is essentially temperature independent, in agreement with experiments. We decompose the selfconsistent harmonic free energy in second-, third-, and fourth-order anharmonic terms. We find that the thirdand fourth-order terms are small. However, treating the third-order term perturbatively on top of the second-order self-consistent harmonic free energy overestimates the energy of the satellite associated with the transverse optical mode. On the contrary, a perturbative treatment on top of the harmonic Hamiltonian breaks down and leads to imaginary phonon frequencies already at $300 \mathrm{~K}$. In the case of SnTe, we describe the occurrence of a ferroelectric transition from the high-temperature $F m \overline{3} m$ structure to the low-temperature $R 3 m$ one. The transition temperature is, however, underestimated with respect to the experimental one. No satellites are present in the SnTe phonon spectra despite a not negligible anharmonic broadening of the zone-center TO mode.
\end{abstract}

DOI: 10.1103/PhysRevB.97.014306

\section{INTRODUCTION}

Thermoelectric materials are appealing for their capability of converting heat into electric power, and vice versa [1,2]. Used in conjunction with clean sources of energy, such as solar radiation, such devices may be an alternative solution for the increasing global energy demand, and other global issues such as global warming [2-5]. The development of efficient thermoelectric devices is linked to a dimensionless quantity called figure of merit, given by:

$$
Z T=\frac{S^{2} \sigma T}{k},
$$

$S$ is the Seebeck coefficient, $T$ the temperature, and $\sigma$ and $k$ are the electronic and thermal conductivities, respectively. The higher is the figure of merit, higher is the efficiency of a thermoelectric device. A possible strategy to increase the figure of merit is to look for systems with small thermal conductivity. In practice, this often means to look for systems with large phonon-phonon scattering, i.e., with dominant anharmonic effects in the phonon spectra. Thus a first step towards design and understanding of new thermoelectric design is the development of a reliable description of anharmonicity in phonon spectra. In addition, as several thermoelectric compounds undergo second-order phase transitions driven by soft modes, a detailed nonperturbative treatment of anharmonicity is crucial to understand the lattice dynamics and thermoelectric efficiency of materials. From a theoretical point of view, several approximations [6-10] have been developed in recent years to tackle this problem and it is finally becoming possible to describe anharmonic effects beyond the perturbative regime $[11,12]$. This is the goal of the present paper, namely the investigation of the reliability of the stochastic self-consistent approximation in describing the vibrational properties of thermoelectrics beyond the harmonic approximation.

Among thermoelectrics, we consider PbTe and SnTe that recently have drawn attention due to some of their interesting properties [13-18]. Both systems have high figures of merit, 
turning them into effective thermoelectrics. Furthermore, at room temperature they have simple NaCl-like structures. The simplicity of their structure and the importance of anharmonic effects to describe their lattice-dynamical properties make them an ideal playground to validate nonperturbative theoretical approaches to the anharmonic problem.

Beside its thermoelectric properties, SnTe displays an intriguing ferroelectric transition at low temperatures. This transition toward a rhombohedral structure occurs when the transverse optical (TO) modes at the Brillouin zone (BZ) center softens during cooling. Past and recent experiments measured different transition temperatures, ranging from $0 \mathrm{~K}$ to values around $100 \mathrm{~K}$. Such a variation in $T_{c}$ is due to the change in the intrinsic doping [19]. On the other hand, PbTe does not undergo a phase transition at low temperature, although it has an incipient ferroelectric nature. Moreover, recent INS experiments reported that $\mathrm{PbTe}$ exhibits a phonon satellite peak close to zone center, a clear fingerprint of strong anharmonicity [16].

From the theoretical point of view, both systems have been studied in the past by using $a b$ initio calculations, as well as molecular-dynamics-based methods [18,20-23]. The majority of them use an nonperturbative approach since previous calculations based on perturbation theory report structural instabilities that are not present in the measurements $[7,20]$. Some of those methods give good agreement with the experiment, specially for PbTe. However, the results may be affected by a conjunction of factors, such as the volume used on the calculations [24]. Furthermore, in all these methods quantum effects were neglected. For PbTe this is a reasonable approximation, however, for SnTe it is not obvious as the temperature of $100 \mathrm{~K}$ corresponds to $8 \mathrm{meV}$ that is approximately the transverse optical phonon energy.

In this paper we present the anharmonic phonon spectra of PbTe and SnTe as function of temperature using the stochastic self-consistent harmonic approximation (SSCHA) [9,10,25-27]. We apply the method to both systems and determine the magnitude of anharmonic effects on vibrational spectra and on the ferroelectric transition.

This paper is presented in the following order. First, in Sec. II we introduce the theoretical background and methodology applied in our calculations. Then we introduce the stochastic self-consistent harmonic approximation (SSCHA) $[9,10,27]$ and, in this framework, the evaluation of the freeenergy Hessian [27]. Section III presents the parameters used in our $a b$ initio calculations. The main results concerning the harmonic and anharmonic phonon dispersions, and comparison between our calculations and experimental data are described in Sec. IV.

\section{THEORY}

We study the lattice dynamic of PbTe and SnTe within the Born-Oppenheimer (BO) approximation. The quantum Hamiltonian for the atoms is defined by the BO potential energy $V(\boldsymbol{R})$. With $\boldsymbol{R}$ we are denoting in component-free notation the quantity $R^{\alpha s}(\boldsymbol{l})$, which is a collective coordinate that completely specifies the atomic configuration of the crystal. The index $\alpha$ denotes the Cartesian direction, $s$ labels the atom within the unit cell, and $\boldsymbol{l}$ indicates the three-dimensional lattice vector. In what follows we will also use a single composite index $a=(\alpha, s, l)$ to indicate Cartesian index, atom index, and lattice vector together. Moreover, in general, we will use bold letters to indicate also other quantities in component-free notation.

In order to take into account quantum effects and anharmonicity at nonperturbative level, we use the stochastic self-consistent harmonic approximation (SSCHA) [9,10,27]. For a given temperature $T$, the method allows us to find an approximate estimation for $F\left(\mathcal{R}^{\alpha s}(\boldsymbol{l})\right)$, the free energy of the crystal as a function of the average atomic position $\mathcal{R}^{\alpha s}(\boldsymbol{l})$ (the centroids). For a given centroid $\mathcal{R}$, the SSCHA free energy is obtained through an auxiliary quadratic Hamiltonian, the SSCHA Hamiltonian $\mathcal{H}_{\mathcal{R}}$. In a displacive second-order phase transition, at high temperature the free energy has minimum in a high symmetry configuration $\boldsymbol{\mathcal { R }}_{\mathrm{hs}}$ but, on lowering temperature, $\boldsymbol{\mathcal { R }}_{\mathrm{hs}}$ becomes a saddle point at the transition temperature $T_{c}$. Therefore, the free-energy Hessian evaluated in $\boldsymbol{\mathcal { R }}_{\mathrm{hs}}$, $\partial^{2} F /\left.\partial \mathcal{R} \partial \mathcal{R}\right|_{\mathcal{R}_{\mathrm{b}}}$, at high temperature is positive definite but it develops one or multiple negative eigendirections at $T_{c}$. The SSCHA free-energy Hessian in a centroid $\mathcal{R}$ can be computed by using the analytic formula (in component-free notation) [27]

$$
\frac{\partial^{2} F}{\partial \mathcal{R} \partial \mathcal{R}}=\boldsymbol{\Phi}+\stackrel{(3)}{\boldsymbol{\Phi}} \boldsymbol{\Lambda}(0)[1-\stackrel{(4)}{\boldsymbol{\Phi}} \boldsymbol{\Lambda}(0)]^{-1} \stackrel{(3)}{\boldsymbol{\Phi}},
$$

with

$$
\begin{aligned}
\boldsymbol{\Phi} & =\left\langle\frac{\partial^{2} V}{\partial \boldsymbol{R} \partial \boldsymbol{R}}\right\rangle_{\rho_{\mathcal{H}_{\mathcal{R}}}}, \\
\stackrel{(3)}{\boldsymbol{\Phi}} & =\left\langle\frac{\partial^{3} V}{\partial \boldsymbol{R} \partial \boldsymbol{R} \partial \boldsymbol{R}}\right\rangle_{\rho_{\mathcal{H}_{\mathcal{R}}}}, \quad \stackrel{(4)}{\boldsymbol{\Phi}}=\left\langle\frac{\partial^{4} V}{\partial \boldsymbol{R} \partial \boldsymbol{R} \partial \boldsymbol{R} \partial \boldsymbol{R}}\right\rangle_{\rho_{\mathcal{H}_{\mathcal{R}}}},
\end{aligned}
$$

where the averages are with respect to the density matrix of the SSCHA Hamiltonian $\mathcal{H}_{\mathcal{R}}$, i.e., $\rho_{\mathcal{H}_{\mathcal{R}}}=e^{-\beta \mathcal{H}_{\mathcal{R}}} / \operatorname{tr}\left[e^{-\beta \mathcal{H}_{\mathcal{R}}}\right]$, and $\beta=\left(k_{b} T\right)^{-1}$ where $k_{b}$ is the Boltzmann constant. In Eq. (2) the value at $z=0$ of the fourth-order tensor $\boldsymbol{\Lambda}(z)$ is used. For a generic complex number $z$ it is defined, in components, by

$$
\begin{aligned}
\Lambda^{a b c d}(z)= & -\frac{1}{2} \sum_{\mu \nu} F\left(z, \omega_{\mu}, \omega_{\nu}\right) \\
& \times \sqrt{\frac{\hbar}{2 M_{a} \omega_{\mu}}} e_{\mu}^{a} \sqrt{\frac{\hbar}{2 M_{b} \omega_{\nu}}} e_{v}^{b} \\
& \times \sqrt{\frac{\hbar}{2 M_{c} \omega_{\mu}}} e_{\mu}^{c} \sqrt{\frac{\hbar}{2 M_{d} \omega_{\nu}}} e_{\nu}^{d},
\end{aligned}
$$

with $M_{a}$ the mass of the atom $a, \omega_{\mu}^{2}$ and $e_{\mu}^{a}$ eigenvalues and corresponding eigenvectors of $D_{a b}^{(S)}=\Phi_{a b} / \sqrt{M_{a} M_{b}}$, respectively, and

$$
\begin{aligned}
F\left(z, \omega_{\nu}, \omega_{\mu}\right)= & \frac{2}{\hbar}\left[\frac{\left(\omega_{\mu}+\omega_{\nu}\right)\left[1+n_{\mathrm{B}}\left(\omega_{\mu}\right)+n_{\mathrm{B}}\left(\omega_{\nu}\right)\right]}{\left(\omega_{\mu}+\omega_{\nu}\right)^{2}-z^{2}}\right. \\
& \left.-\frac{\left(\omega_{\mu}-\omega_{\nu}\right)\left[n_{\mathrm{B}}\left(\omega_{\mu}\right)-n_{\mathrm{B}}\left(\omega_{\nu}\right)\right]}{\left(\omega_{\mu}-\omega_{\nu}\right)^{2}-z^{2}}\right],
\end{aligned}
$$

where $n_{\mathrm{B}}(\omega)=1 /\left(e^{\beta \hbar \omega}-1\right)$ is the bosonic occupation number. Evaluating through Eq. (2) the free-energy Hessian in $\boldsymbol{\mathcal { R }}_{\mathrm{hs}}$ and studying its spectrum as a function of temperature, we 
can predict the occurrence of a displacive second-order phase transition and estimate the relative $T_{c}$. Equivalently, we can analyze lattice instabilities by studying the softening in the eigenvalues of

$$
D_{a b}^{(F)}=\frac{1}{\sqrt{M_{a} M_{b}}} \frac{\partial^{2} F}{\partial \mathcal{R}^{a} \partial \mathcal{R}^{b}},
$$

which can be considered an anharmonic, temperaturedependent generalization of the standard harmonic dynamical matrix. Moreover, since we are considering a crystal, we can take advantage of the lattice translation symmetry and Fourier transform with respect to the lattice indexes. Therefore, since there are two atoms in the unit cell of PbTe and SnTe, we actually calculate the eigenvalues the sixth-order square matrix $D_{\alpha s \beta t}^{(F)}(\boldsymbol{q})$ in different $\boldsymbol{q}$ points of the Brillouin zone. The magnitude of the different terms comprising Eq. (2) is discussed in Appendix A. In particular it is shown that, for the applications considered in the present paper, the term $\stackrel{(4)}{\Phi} \boldsymbol{\Lambda}(0)$ is negligible with respect to the identity matrix [or, using the Appendix's notation, $\langle D 4 V\rangle$ is negligible].

As shown in Ref. [27], in the context of the SCHA it is possible to formulate an ansatz in order to give an approximate expression of the one-phonon Green's function $\boldsymbol{G}(z)$ for the variable $\sqrt{M_{a}}\left(R^{a}-\mathcal{R}_{\mathrm{hs}}^{a}\right)$ :

$$
\boldsymbol{G}^{-1}(z)=z^{2} \mathbb{1}-\boldsymbol{M}^{-\frac{1}{2}} \boldsymbol{\Phi} \boldsymbol{M}^{-\frac{1}{2}}-\boldsymbol{\Pi}(z) .
$$

Here $M_{a b}=\delta_{a b} M_{a}$ is the mass matrix and $\Pi(z)$ is the SSCHA self-energy, which is given by

$$
\Pi(z)=M^{-\frac{1}{2}} \stackrel{(3)}{\boldsymbol{\Phi}} \boldsymbol{\Lambda}(z)[\mathbb{1}-\stackrel{(4)}{\boldsymbol{\Phi}} \boldsymbol{\Lambda}(z)]^{-1} \stackrel{(3)}{\boldsymbol{\Phi}} \boldsymbol{M}^{-\frac{1}{2}}
$$

Notice that $\boldsymbol{G}^{-1}(0)=-\boldsymbol{D}^{(F)}$, as it must be. We extend the negligibility of the static $\stackrel{(4)}{\Phi} \Lambda(0)$ to the generic dynamical case. That allows us to neglect $\stackrel{(4)}{\boldsymbol{\Phi}} \boldsymbol{\Lambda}(z)$ and reduce the SSCHA selfenergy to the so-called bubble self-energy, namely:

$$
\Pi(z) \approx \Pi^{(B)}(z)=M^{-\frac{1}{2}} \stackrel{(3)}{\boldsymbol{\Phi}} \boldsymbol{\Lambda}(z) \stackrel{(3)}{\boldsymbol{\Phi}} \boldsymbol{M}^{-\frac{1}{2}}
$$

Again, we take advantage of lattice translation symmetry and Fourier transform with respect to the lattice indexes. Moreover, we neglect the mixing between different phonon modes and assume that the self-energy $\boldsymbol{\Pi}(\boldsymbol{q}, z)$ is diagonal in the basis of the eigenvectors $e_{\mu}^{\alpha s}(\boldsymbol{q})$ of $D_{\alpha s \beta t}^{(S)}(\boldsymbol{q})$. Thus we consider the quantity

$$
\Pi_{\mu}(\boldsymbol{q}, \omega)=\sum_{\alpha s, \beta t} e_{\mu}^{\alpha s}(-\boldsymbol{q}) \Pi_{\alpha s \beta t}\left(\boldsymbol{q}, \omega+i 0^{+}\right) e_{\mu}^{\beta t}(\boldsymbol{q}) .
$$

From the one-phonon Green's function we obtain the corresponding spectral function $-2 \operatorname{Tr} \operatorname{Im} \boldsymbol{G}\left(\boldsymbol{q}, \omega+i 0^{+}\right)$. Peaks in this quantity, as a function of $\omega$, signal the presence of collective vibrational excitations (phonons) having certain energies, as they can be probed with inelastic scattering experiments. We find convenient to multiply the spectral function by the factor $\omega / 2 \pi, \sigma(\boldsymbol{q}, \omega)=-\omega \operatorname{Tr} \operatorname{Im} \boldsymbol{G}\left(\boldsymbol{q}, \omega+i 0^{+}\right) / \pi$. In this way the integral, on the real axis, of $\sigma(\omega, \boldsymbol{q})$ with respect to $\omega$ is equal to the number of modes ( $\mathrm{six}$, in this case). It is

$$
\begin{aligned}
& \sigma(\boldsymbol{q}, \omega) \\
& =\frac{1}{\pi} \sum_{\mu} \frac{-\omega \operatorname{Im} \Pi_{\mu}(\boldsymbol{q}, \omega)}{\left(\omega^{2}-\omega_{\mu \boldsymbol{q}}^{2}-\operatorname{Re} \Pi_{\mu}(\boldsymbol{q}, \omega)\right)^{2}+\left(\operatorname{Im} \Pi_{\mu}(\boldsymbol{q}, \omega)\right)^{2}} .
\end{aligned}
$$

The square phonon frequencies corrected by the self-energy, $\Omega_{\mu q}^{2}$, are then obtained as

$$
\Omega_{\mu \mathbf{q}}^{2}=\omega_{\mu \mathbf{q}}^{2}+\operatorname{Re} \Pi_{\mu}\left(\mathbf{q}, \omega_{\mu \mathbf{q}}\right)
$$

\section{METHODS}

We perform density-functional theory (DFT) calculations using the QUANTUM-ESPRESSO package [28]. For both systems the exchange-correlation interaction is treated with the Perdew-Burke-Ernzerhof (PBE) generalized gradient approximation [29]. To describe the interaction between electrons and ions, we use norm-conserving pseudopotentials [30] for $\mathrm{PbTe}$, and PAW [31] pseudopotentials for SnTe. In both case the semicore $4 d$ states in valence are included for Te and $\mathrm{Sn}$, and $5 d$ for $\mathrm{Pb}$. Electronic wave functions are expanded in a plane-wave basis with kinetic energy cutoffs of 65 Ry and 28 Ry for scalar relativistic pseudopotentials, for $\mathrm{PbTe}$ and $\mathrm{SnTe}$, respectively. Integrations over the Brillouin zone (BZ) are performed using a uniform grid of $8 \times 8 \times 8 \mathbf{k}$ points for $\mathrm{PbTe}$, and a denser grid of $12 \times 12 \times 12 \mathbf{k}$ for SnTe. Particular care must be taken in converging $\mathrm{SnTe}$ with respect to $\mathbf{k}$ points as the depth of the potential well as a function of phonon displacements is strongly dependent on the sampling. Previous calculations [21] carried out with smaller samplings are found to be underconverged (see Appendix B for convergence tests). Born effective charges calculated via density-functional perturbation theory (DFPT) are included for PbTe calculations only. For both PbTe and SnTe we consider the high-temperature rock-salt structure, and the PBE optimized lattice parameters of $6.55 \AA$ and $6.42 \AA$, respectively. The effect of the thermal expansion is discussed in Appendix C.

Harmonic phonon frequencies are calculated within the DFPT [32] as implemented in QUANTUM-ESPRESSO. We investigate the $2 \times 2 \times 2$, and then, the $4 \times 4 \times 4$ q-point grids for both systems. Fourier interpolation is used to obtain the phonon dispersion along high symmetry lines.

To calculate the anharmonic renormalized phonons we use the SSCHA $[9,10,27]$. The trial Hamiltonian is minimized in a supercell. This minimization precess requires the energies and forces acting on a supercell for a set of random configurations generated by the trial density matrix. Those elements have been calculated on $2 \times 2 \times 2$ and $4 \times 4 \times 4$ supercells using the same parameters for the harmonic DFPT calculations. The number of random configurations we use is of the order of 1000 . The difference between harmonic and anharmonic dynamical matrices is interpolated to a $14 \times 14 \times 14$ supercell for SnTe. For $\mathrm{PbTe}$ no interpolation is needed since the $4 \times 4 \times 4$ supercell is converged and adequate to describe the experimental results. 


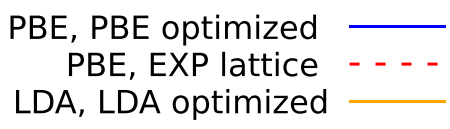

PbTe Harmonic Phonons

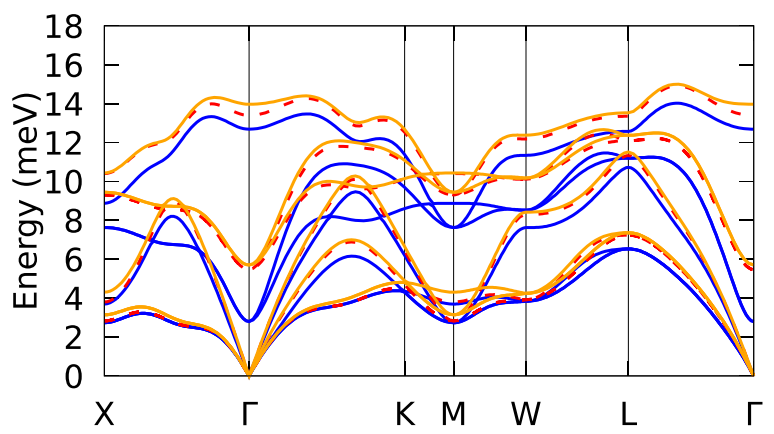

SnTe Harmonic Phonons

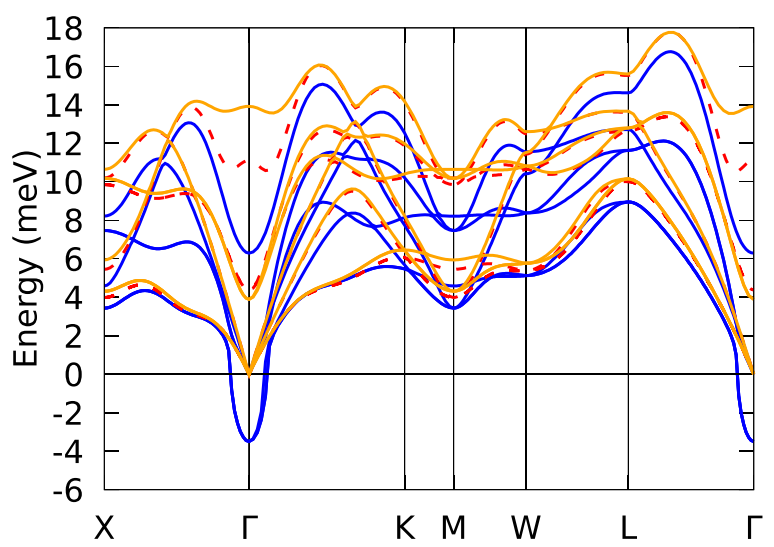

FIG. 1. Harmonic phonons dispersion of PbTe and SnTe for different lattice constants and functionals. LO/TO splitting is included in the calculation. For the experimental lattice parameter (red lines) both systems do not present negative frequencies. For the PBE optimized lattice parameter (blue lines), the SnTe phonon spectra has negative frequencies on $\Gamma$, indicating a structural instability. The instability disappears if the experimental or local density approximation (LDA) volumes are used. The TO modes at $\Gamma$ exhibit an strong dependence on the volume.

\section{RESULTS AND DISCUSSION}

\section{A. Harmonic phonon dispersion}

In ferroelectrics and thermoelectrics the phonon spectra strongly depends on the volume used in the calculations [24]; we first investigate the dependence of the harmonic phonon spectra on the lattice parameters. For this reason in Fig. 1 we calculate harmonic phonon dispersions within DFPT for PbTe and SnTe using (i) the PBE functional on top of the PBE geometry at zero temperature $\left[a_{\mathrm{PBE}}(\mathrm{PbTe})=6.55 \AA, a_{\mathrm{PBE}}(\mathrm{SnTe})=\right.$ $6.42 \AA]$, (ii) the PBE functional on top of the experimental $[33,34]$ geometry $\left[a_{\exp }(\mathrm{PbTe})=6.46 \AA\right.$ and $a_{\exp }(\mathrm{SnTe})=$ $6.32 \AA]$, and (iii) the local density approximation functional $(\mathrm{LDA})$ on top of the LDA geometry $\left[a_{\mathrm{LDA}}(\mathrm{PbTe})=6.39 \AA\right.$ and $a_{\mathrm{LDA}}(\mathrm{SnTe})=6.24 \AA$, respectively. The experimental parameter is smaller (larger) than the theoretical PBE (LDA) one, as if the system were experiencing a finite negative (positive) pressure. As expected, the results are strongly volume dependent. In the case of $\mathrm{PbTe}$, the use of the PBE functional on top of the experimental geometry hardens all the PBE phonons on top of the PBE geometry, but the hardening is particularly large for the transverse optical (TO) mode at zone center that is shifted from $3.17 \mathrm{meV}$ to $6.19 \mathrm{meV}$. The LDA functional on top of the LDA geometry gives even harder phonon frequencies than PBE on top of the experimental geometry. However, in all cases, the harmonic phonon frequencies are positive and no structural instability is detected in $\mathrm{PbTe}$, in agreement with experiments. $\mathrm{PbTe}$ is usually referred to as an incipient ferroelectric because of the softness of the TO phonon mode. It is important to underline that in the case of $\mathrm{PbTe}$ the experiments $[15,16]$ find a clear LO/TO splitting. We thus included this effect in our harmonic calculation.

At ambient pressure SnTe undergoes a phase transition in the 30-100 K temperature range. At low temperature the crystal symmetry changes from cubic $(F m \overline{3} m)$ to rhombohedral $(R 3 m)$. The distortion is a displacive phase transition involving a small dimerization in the unit cell [35]. The distortion is compatible with a phonon instability at zone center. Real samples of SnTe are nonstoichiometric and the ferroelectric transition temperature strongly depends on the number of holes present in the system. It is approximately $100 \mathrm{~K}$ for hole concentrations of the order of $1 \times 10^{20} \mathrm{~cm}^{-3}$ and decreases to approximately $30 \mathrm{~K}$ for ten times larger hole concentrations. At these large doping is is not clear if the LO/TO splitting needs to be included in the calculation. For this reason, for the case of SnTe we perform calculations both with and without LO/TO splitting.

The dependence of the harmonic calculation on volume is stronger in the case of SnTe. The ferroelectric transition (imaginary TO phonon at zone center) is present when using the PBE functional on top of the PBE geometry, while it disappears if the experimental or LDA geometries are used. This is consistent with the disappearing of the ferroelectric transition at finite pressure $[36,37]$ and again underlines the critical role of the volume used in the calculation of phonon spectra in ferroelectrics and thermoelectrics [38]. Finally, as SnTe is substantially doped, the electronic screening is a compromise between a metallic screening and the poor screening of a polar insulator. For this reason, in Appendix D, we investigate the role of metallic screening by considering a hole doped $\mathrm{SnTe}$ and by using the virtual crystal approximation (namely by changing the number of electrons in the simulation and by introducing a compensating jellium background). We find that globally the effects of metallic screening are small, except at very small momenta.

In the rest of the paper we then neglect metallic screening, consider stoichiometric SnTe and we use the PBE optimized lattice parameter at $T=0 \mathrm{~K}$ both for $\mathrm{PbTe}$ and $\mathrm{SnTe}$ in all calculations. For $\mathrm{SnTe}$, as we are interested in the temperatures below $100 \mathrm{~K}$, we neglect the effects of the thermal expansion.

\section{B. Anharmonic phonons \\ 1. Lead telluride (PbTe)}

In Fig. 2 we compare the $\mathrm{PbTe}$ phonon dispersion curves at $300 \mathrm{~K}$ obtained by the SSCHA to INS experimental data 


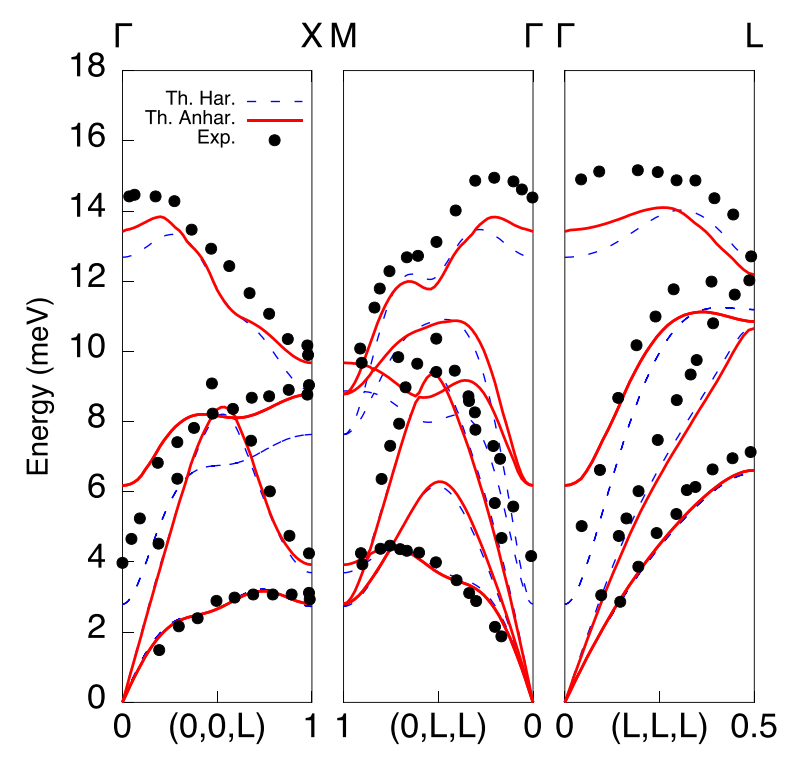

FIG. 2. PbTe harmonic (dashed lines) and anharmonic (solid lines) phonon dispersion curves at $300 \mathrm{~K}$ compared with INS experiments [15] at $300 \mathrm{~K}$ (black dots). The anharmonic phonon dispersion $\left(\Omega_{\mu \mathbf{q}}\right)$ is obtained from Eq. (12) and includes the contribution from the bubble self-energy.

obtained by Cochran et al. [15]. Our calculated curves are in good agreement with experimental results. We obtain a higher value than the experiments for the TO modes at zone center is consistent to newer observations of a double peak in this region [16]. Previous calculations [23] found a good agreement for the lower-energy TO mode at $\Gamma$, however, not obtaining a good description of the high-energy phonon branches. More recent INS measurements [16] have suggested the presence of a strong temperature-dependent phonon satellite close to $\Gamma$ originated from the TO mode. Furthermore, as the temperature increases, the TO band is shifted to higher energies and the crossing between LA and TO phonon bands along the $\Gamma \mathrm{X}$ direction becomes less evident.

In order to determine if the SSCHA approximation can describe phonon satellites and to investigate the temperature dependence of the crossing, the phonon self-energy is calculated performing Fourier interpolation over a denser $40 \times 40 \times 40$ phonon momentum grid and a $0.2 \mathrm{meV}$ broadening to regularize the integral in Eq. (5). Figure 3 shows our calculated anharmonic phonon dispersion versus the spectral function for $\mathrm{PbTe}$ obtained by using the SCHA self-energy. We also show, with pink dots, the energy of the TO phonon and of its satellite as measured in INS experiments detailed in Ref. [16].

Both the satellite and the crossing of LA and TO bands at $300 \mathrm{~K}$ are well described by our methodology. Moreover, the energies of the TO peaks at $\Gamma$ obtained by the SSCHA are compatible with the observed values. The presence of these features were also investigated in the literature using different methods. In particular, nonperturbative methods such as the temperature-dependent effective potential technique (TDEP) $[7,20]$ were able to obtain similar results. Finally, we find that the satellite energy depends weakly on temperature (not shown), although it substantially broadens as a function of temperature.

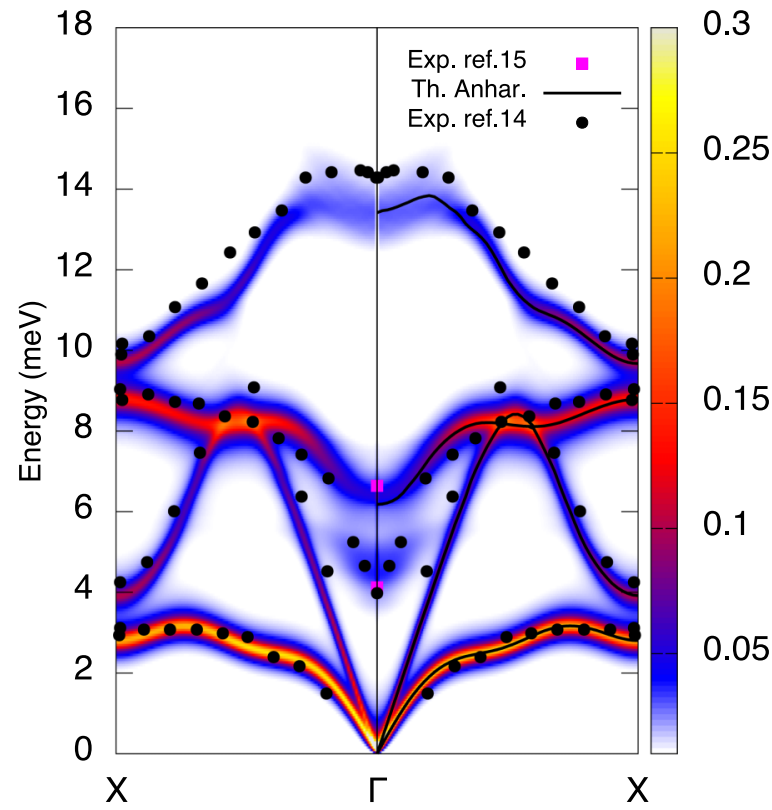

FIG. 3. PbTe Spectral function at $300 \mathrm{~K}$ calculated along the $X-\Gamma-X$ path (color map). Solid lines denote the anharmonic phonon dispersion curves, black dots denote the experimental data from Ref. [15] and pink squares denote the experimental values for the peaks at the zone center from more recent experiments described in Ref. [16]. The color code is determined by the value of $\sigma(\boldsymbol{q}, \omega)$ in Eq. (11).

\section{Tin telluride (SnTe)}

Calculations for SnTe are reported in Fig. 4 where the comparison between the phonon spectra obtained via SSCHA

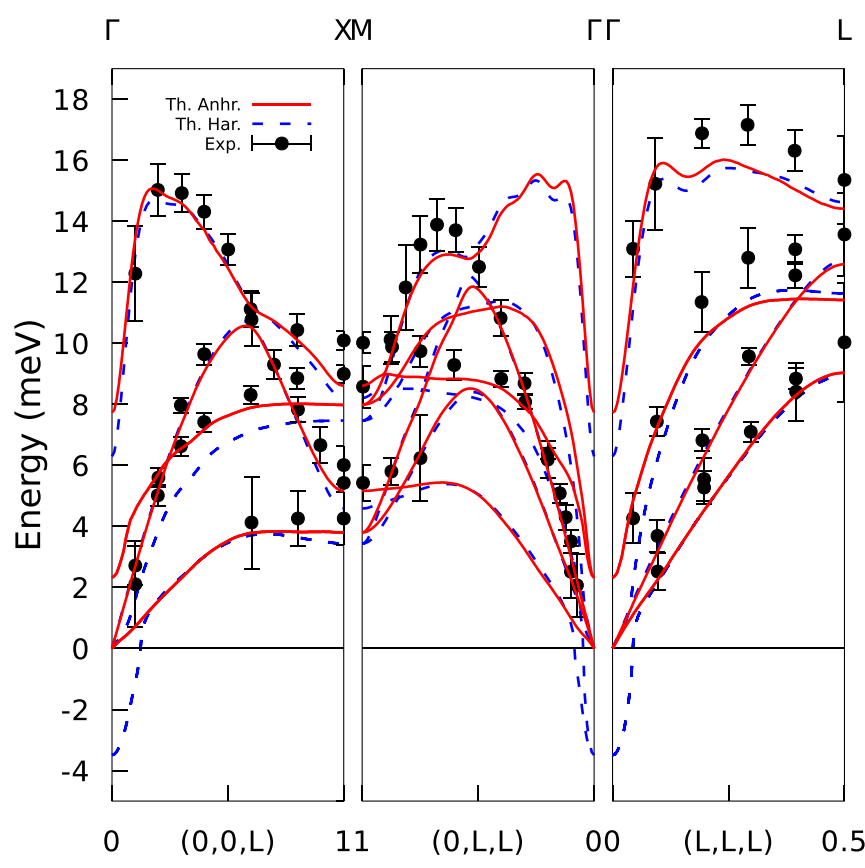

FIG. 4. Harmonic (dashed lines) and anharmonic (solid lines) phonon dispersion relations of $\mathrm{SnTe}$ at $100 \mathrm{~K}$ (red lines). The spectral weight is calculated on a $4 \times 4 \times 4$ supercell. The anharmonic phonon dispersion $\left(\Omega_{\mu \mathbf{q}}\right)$ is obtained from Eq. (12) and includes the contribution form the bubble self-energy. 
at $T=100 \mathrm{~K}$ and recent IXS experiments [18] at $T=75 \mathrm{~K}$ is shown. Even, if this system was studied theoretically before, using methods such as the TDEP and self-consistent ab initio lattice dynamics (SCAILD) $[8,18]$, the calculations focused on higher temperatures. Our calculated anharmonic dispersion curves present the main features of the experimental data for all investigated high symmetry directions along the BZ. Overall, we find a good agreement with experimental data. In Fig. 5 we compare the SnTe calculated anharmonic phonon dispersion and the spectral function for SnTe obtained by using the SCHA self-energy and compared with experiments. The calculation of the SCHA self energy is carried out on a $4 \times 4 \times 4$ supercell. The linewidth is interpolated over $80 \times 80 \times 80$ phonon momentum grids and a broadening of $0.2 \mathrm{meV}$ is used to regularize the integral in Eq. (5). Contrary to the case of $\mathrm{PbTe}$, we do not find the occurrence of any satellite
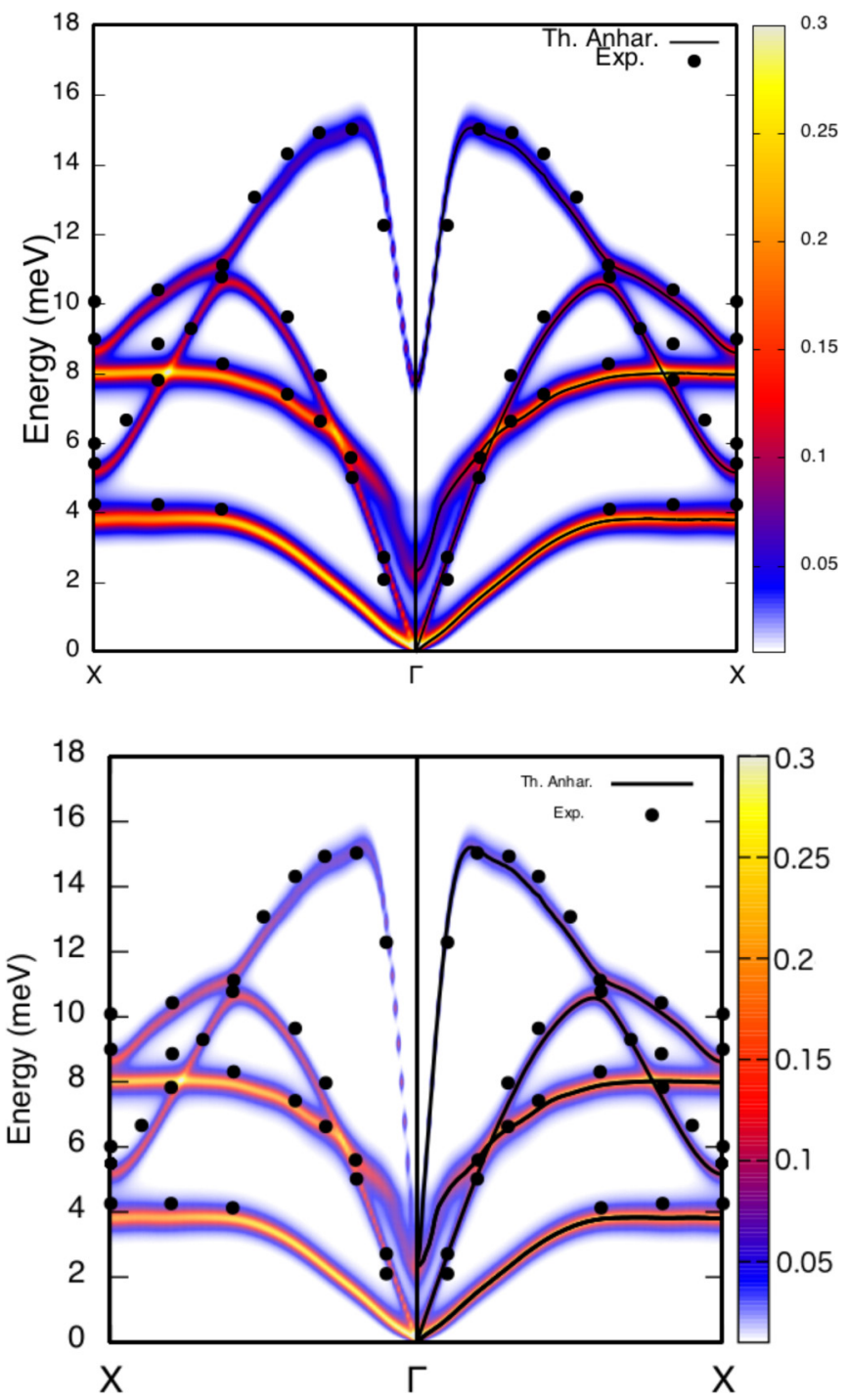

FIG. 5. SnTe spectral function at $100 \mathrm{~K}$ calculated along the $X-\Gamma-X$ path (color map) with (top) and without (bottom) inclusion of $\mathrm{LO} / \mathrm{TO}$ splitting in the calculation. Solid lines denote the anharmonic phonon dispersion curves, black dots denote the experimental data from Ref. [18] at $75 \mathrm{~K}$ (black dots). The color code is determined by the value of $\sigma(\boldsymbol{q}, \omega)$ in Eq. (11).

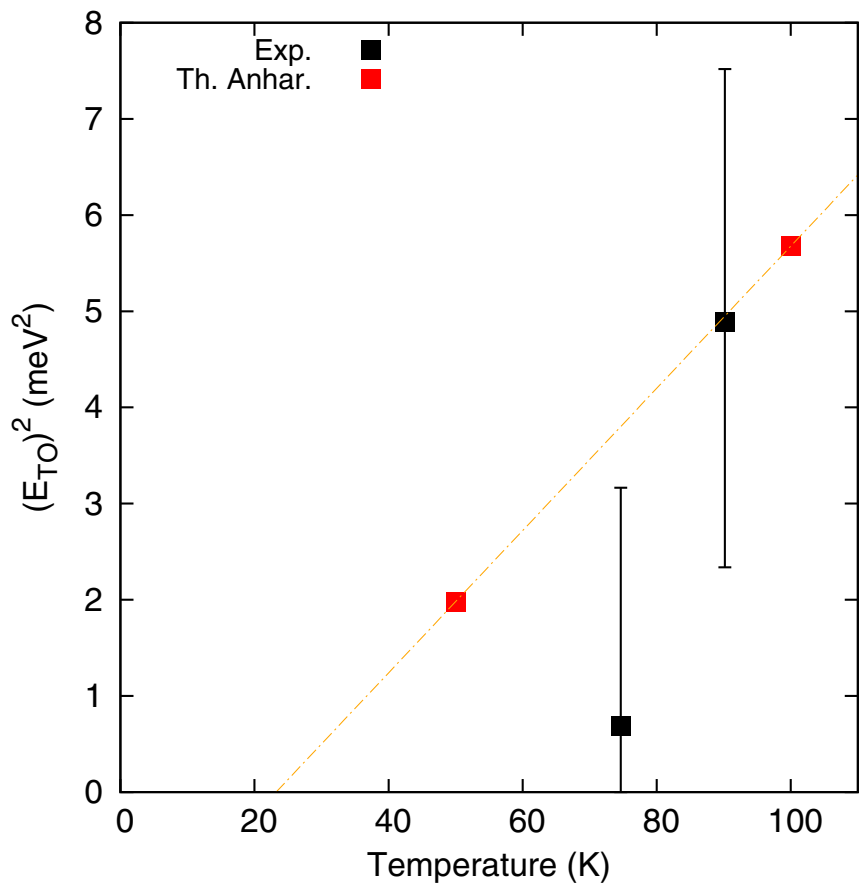

FIG. 6. Energy square of the TO phonons on the zone center plotted against temperature. The red squares denote the results obtained by using Eq. (6), and black squares results from O'Neill et al. [18]. The linear extrapolation indicates that the mode softens to zero energy towards $T_{c} \approx 23 \mathrm{~K}$ for our calculations. In samples at very low doping, the ferroelectric transition can be as high as $100 \mathrm{~K}$.

despite a substantial anharmonic broadening of low-energy modes.

In order to study the second-order structural phase transition in SnTe we evaluate the energy squared of the TO modes at $\Gamma$ as a function of temperature $T$. Our data with the inclusion of anharmonicity are consistent with a ferroelectric transition at $\approx$ $23 \mathrm{~K}$. However, this value should be taken with care as the theoretical calculations are limited by the error in the knowledge of the exchange correlation functional that leads to a big variation in the equilibrium volume. On the other hand, experimentally, the transition temperature of SnTe is strongly dependent on sample doping, varying from $0 \mathrm{~K}$ to around $100 \mathrm{~K}$ for different carriers concentrations. Figure 6 compares our results for the energy squared of the TO mode with recent IXS [18] experiments.

\section{CONCLUSIONS}

We applied a novel technique [27] based on the stochastic self-consistent harmonic approximation capable of investigating phase transitions via the calculation of the Hessian of the free energy. We have studied the temperature-dependent anharmonic phonon spectra of PbTe and SnTe. We found a strong dependence of vibrational properties on the exchangecorrelation functional used in the calculation and on the corresponding equilibrium volume. By using the PBE [29] functional with the theoretical equilibrium volume, we find very good agreement with experimental INS spectra. The SSCHA is not only capable of describing single-particle spectra, but also many-body features such as phonon satellites are 
correctly explained. Finally, we describe the occurrence ferroelectric transition in SnTe from the high- $T F m \overline{3} m$ structure to the low- $T R 3 m$ one. The value of the ferroelectric critical temperature is found to be strongly dependent in the volume used in the calculations, consequently, on the exchange-correlation functional.

\section{ACKNOWLEDGMENTS}

M.C. and F.M. acknowledge support from the Graphene Flagship, PRACE for awarding us access to resource on Marenostrum at BSC and the computer facilities provided by CINES, IDRIS, and CEA TGCC (Project A0010 907320). I.E. acknowledges financial support from the Spanish Ministry of Economy and Competitiveness (FIS2016-76617-P). G.R. acknowledges support from the CAPES foundation-Brazil (Grant No. 99999.009465/2013-08).

\section{APPENDIX A: MAGNITUDE OF $\langle D 3 V\rangle$ AND $\langle D 4 V\rangle$ TERMS}

In order to have a better visualization of the different terms of Eq. (2) one can rewrite it as:

$$
\frac{\partial^{2} F}{\partial \mathcal{R} \partial \mathcal{R}}=\boldsymbol{\Phi}+\stackrel{(3)}{\boldsymbol{\Phi}} \boldsymbol{\Lambda}(0) \stackrel{(3)}{\boldsymbol{\Phi}}+\stackrel{(3)}{\boldsymbol{\Phi}} \boldsymbol{\Lambda}(0) \boldsymbol{\Theta} \Lambda(0) \stackrel{(3)}{\boldsymbol{\Phi}}
$$
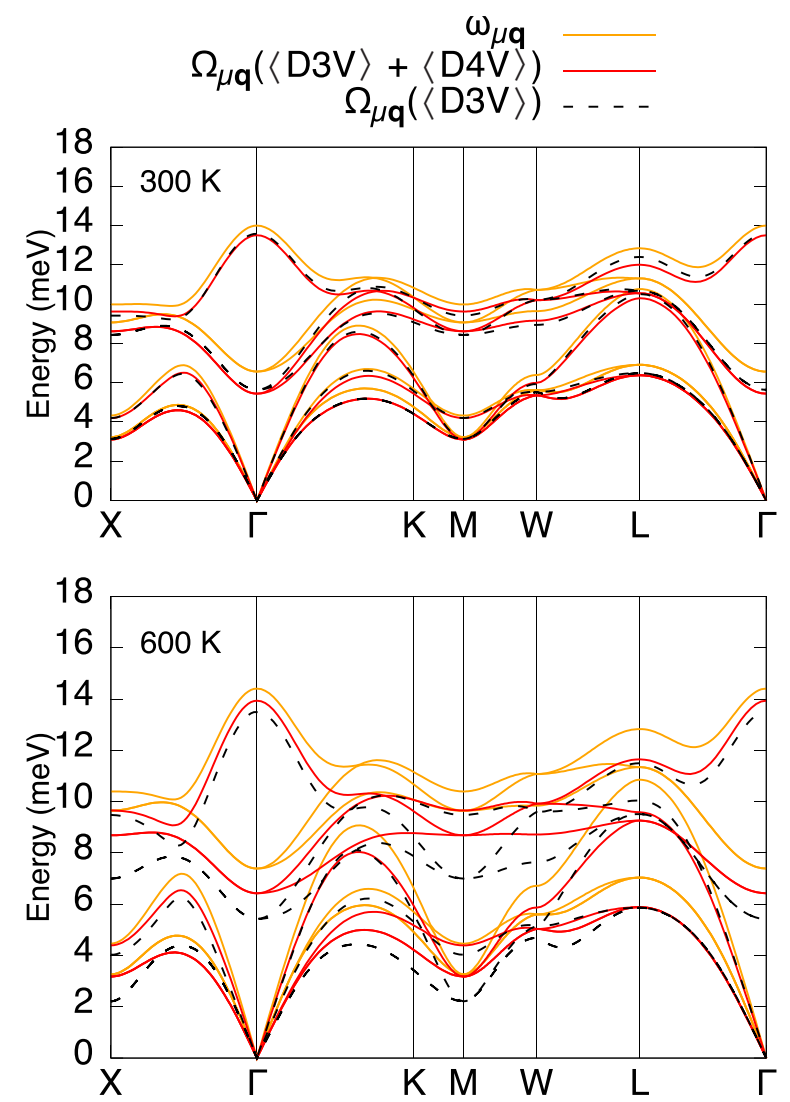

FIG. 7. Anharmonic phonon dispersion curves for PbTe at $300 \mathrm{~K}$ and $600 \mathrm{~K}$. Orange lines denote calculations neglecting the bubble and superior order terms, $\left(\omega_{\mu \mathbf{q}}\right)$. Black dashed lines represent the calculations including $\langle D 3 V\rangle$, while red lines includes the full expression, $\langle D 3 V\rangle+\langle D 4 V\rangle,\left(\Omega_{\mu \mathbf{q}}\right)$. The phonon frequencies are obtained using Eq. (12) in the static limit, namely by using $\Pi_{\mu}(\mathbf{q}, 0)$. where

$$
\boldsymbol{\Theta}=[\mathbb{1}-\stackrel{(4)}{\boldsymbol{\Phi}} \boldsymbol{\Lambda}(0)]^{-1} \stackrel{(4)}{\boldsymbol{\Phi}}
$$

For simplicity, we define

$$
\begin{gathered}
\langle D 3 V\rangle=\stackrel{(3)}{\boldsymbol{\Phi}} \boldsymbol{\Lambda}(0) \stackrel{(3)}{\boldsymbol{\Phi}} \\
\langle D 4 V\rangle=\stackrel{(3)}{\boldsymbol{\Phi}} \boldsymbol{\Lambda}(0) \boldsymbol{\Theta} \Lambda(0) \stackrel{(3)}{\boldsymbol{\Phi}} .
\end{gathered}
$$

To investigate the different terms in Eq. (A1), and in particular the mutual role of $\langle D 3 V\rangle$ and $\langle D 4 V\rangle$ we perform SSCHA runs, on a $2 \times 2 \times 2$ supercell, for different temperatures; 300 and $600 \mathrm{~K}$ for $\mathrm{PbTe}$, and 50 and $100 \mathrm{~K}$ for SnTe.

Figures 7 and 8 compare the contribution of $\langle D 3 V\rangle$ and $\langle D 4 V\rangle$ to the phonon frequencies. Our calculations show that for $\mathrm{PbTe}$ the $\langle D 4 V\rangle$ term is negligible below $300 \mathrm{~K}$ while it is somewhat more relevant at $600 \mathrm{~K}$. For SnTe in the temperature range studied in this work the $\langle D 4 V\rangle$ term is also negligible.
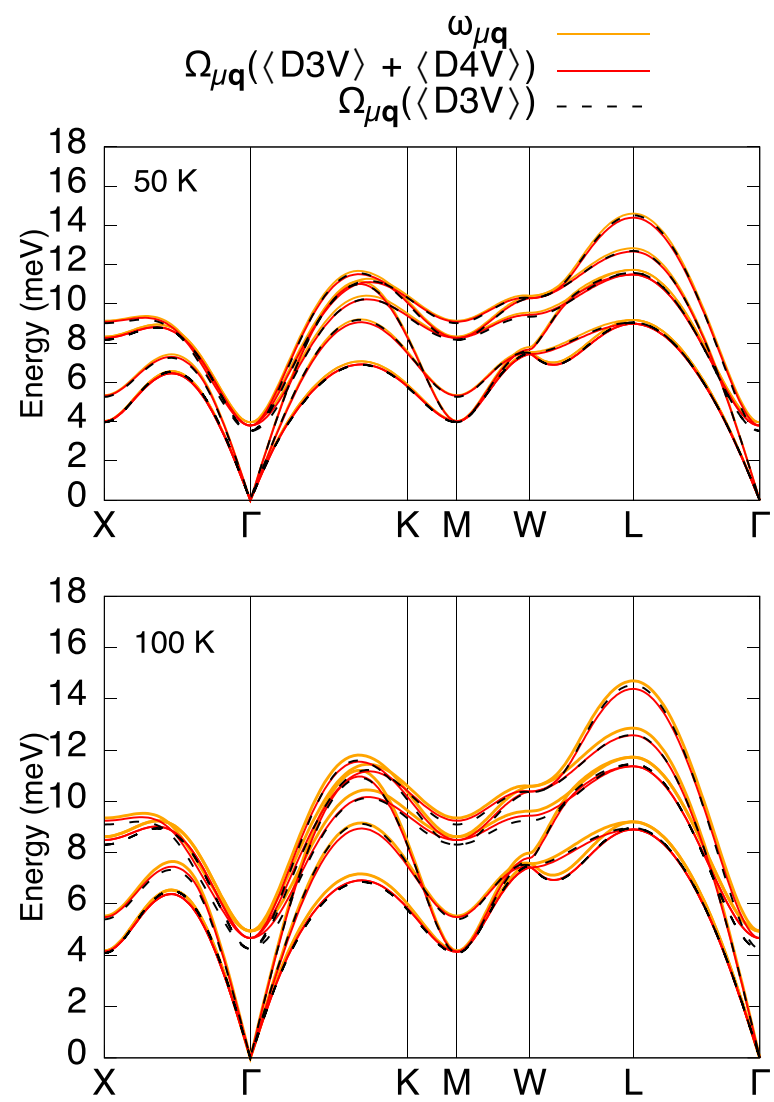

FIG. 8. SSCHA runs for SnTe at $50 \mathrm{~K}$ and $100 \mathrm{~K}$. Orange lines denote calculations neglecting the bubble and superior order terms, $\left(\omega_{\mu \mathbf{q}}\right)$. Black dashed lines represent the calculations including $\langle D 3 V\rangle$, while red lines includes the full expression, $\langle D 3 V\rangle+\langle D 4 V\rangle,\left(\Omega_{\mu \mathbf{q}}\right)$. The phonon frequencies are obtained using Eq. (12) in the static limit, namely by using $\Pi_{\mu}(\mathbf{q}, 0)$. The calculations have been carried out without LO/TO splitting. 

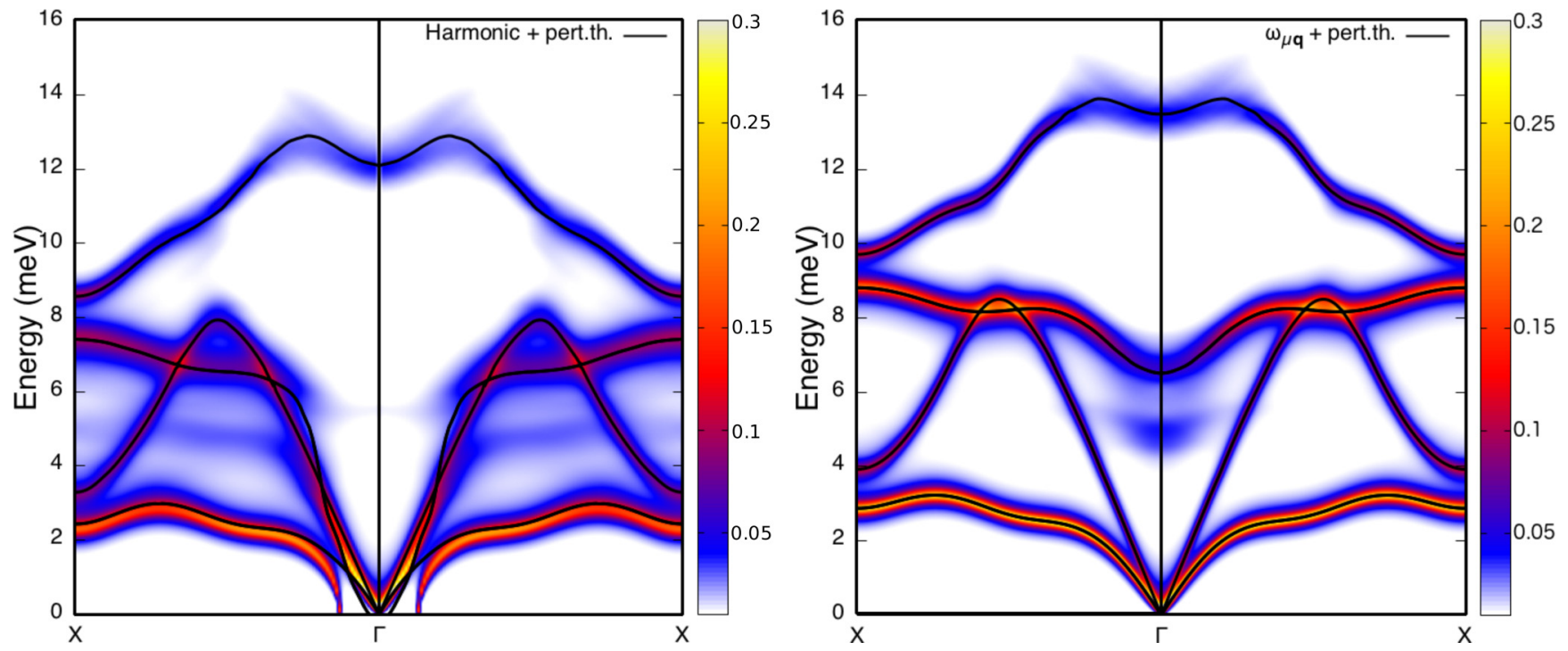

FIG. 9. Validity of perturbation theory in PbTe. Left: third-order anharmonicity is treated perturbatively on top of the harmonic spectrum of Fig. 1. Note the imaginary phonon frequency at zone center at $300 \mathrm{~K}$. Right: third-order anharmonicity treated perturbatively on top of the second order expansion of the SSCHA free energy, namely on top of the phonon frequencies $\omega_{\nu \mathbf{q}}$ (or equally on top of the auxiliary self-consistent harmonic matrix $\Phi)$.

As a consequence, in these temperature regions the Hessian of the free energy is entirely determined by the $\boldsymbol{D}^{(S)}$ matrix and the so-called bubble term $\langle D 3 V\rangle$. This analysis justifies why we neglect the $\langle D 4 V\rangle$ term in the calculations for larger supercells.

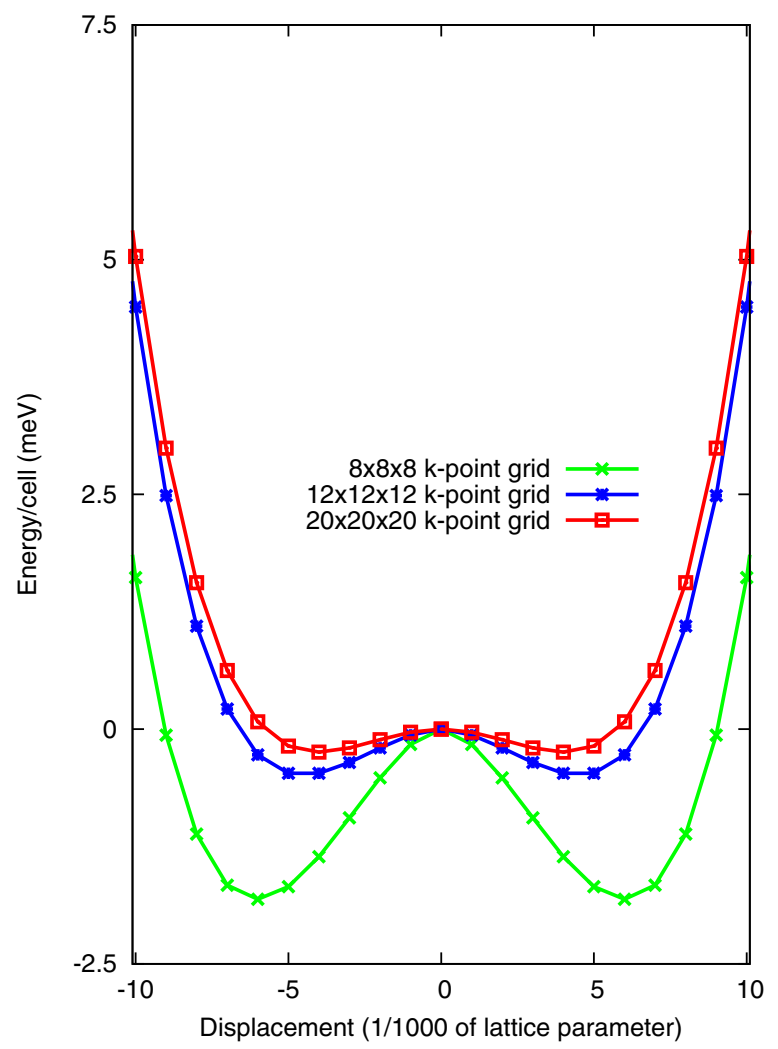

FIG. 10. Frozen phonon potential for SnTe as a function of the $k$-point grid.
Having determined the smallness of $\langle D 4 V\rangle$ we proceed toward larger supercell calculations. By using an empirical potential fitted on the SSCHA configurations we check the convergence with respect to supercell size (see Appendix E). We found that the use of a $4 \times 4 \times 4$ supercell leads to converged phonon frequencies. So we use this supercell to carry out our first-principles calculations.

Given the fact that the $\langle D 3 V\rangle$ and $\langle D 4 V\rangle$ terms are small, it is instructive to see if they can be treated perturbatively. If perturbation theory is started on top of the initial harmonic Hamiltonian, namely that leading to the phonon frequencies in Fig. 1, then perturbation theory breaks down and imaginary phonon frequencies are obtained at $T=300 \mathrm{~K}$, as shown in Fig. 9. This is because the third-order anharmonic correction is so large that the system becomes unstable. On the contrary, if perturbation theory is carried out on top of the second order expansion of the SSCHA free energy, namely on top of the phonon frequencies $\omega_{\nu \mathbf{q}}$ (or equally on top of the free energy $\Phi)$, then the third-order term becomes substantially smaller and perturbation theory is well defined. In practice this means that higher-order anharmonic interactions renormalize third-order anharmonicity. Even in this case, however, the energy of the phonon satellite is slightly overestimated both with respect to experiments and with respect to the more complete SSCHA nonperturbative treatment.

\section{APPENDIX B: K-POINTS SAMPLING IN SnTe}

The convergence of DFT calculations for SnTe is quite tricky. Even if the difference on the total energy of the $F m \overline{3} m$ structure between the $8 \times 8 \times 8$ and $20 \times 20 \times 20 k$-point grids is of the order of $0.8 \mathrm{meV} /$ atom, upon distortion towards the $R 3 \mathrm{~m}$ structure, the depth of the potential well differs of $1.55 \mathrm{meV} /$ cell. Thus, using a smaller $k$-point grid, as done previous calculations [21], substantially overestimates the ferroelectric instability, as shown in Fig. 10. For this reason 

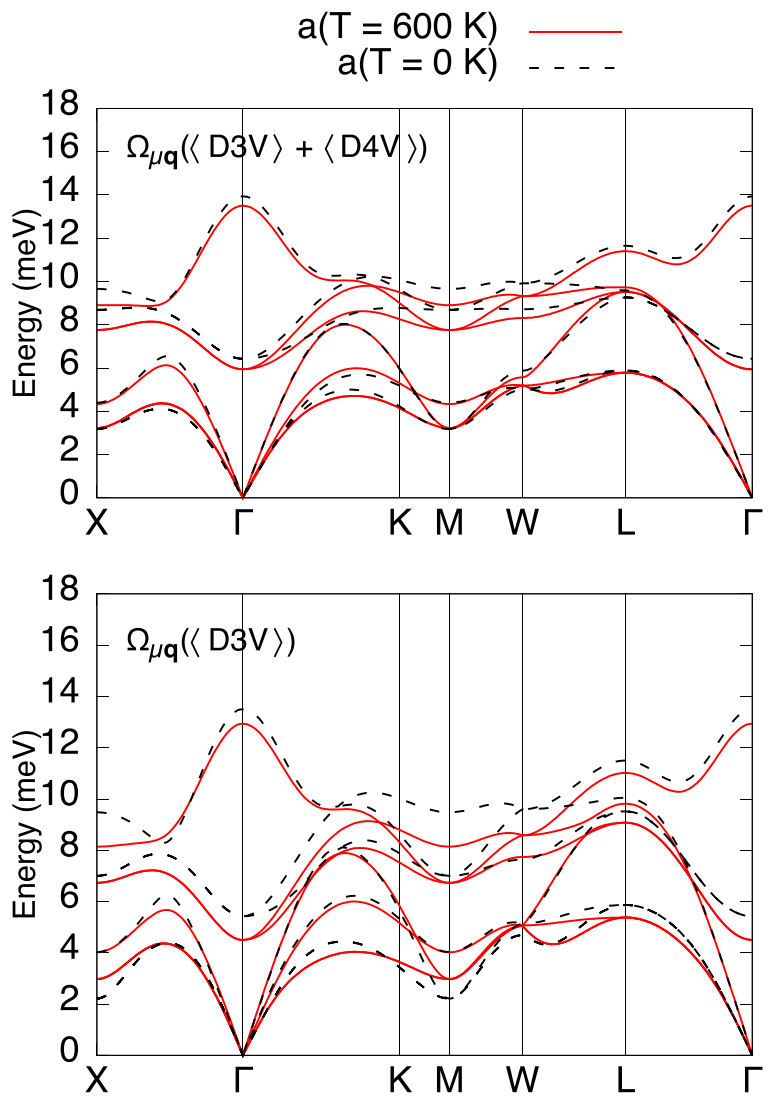

FIG. 11. Anharmonic phonon spectra for the PBE at $600 \mathrm{~K}$ lattice parameter versus the $\mathrm{PBE}$ at $0 \mathrm{~K}$ case. It is noticeable that the difference is larger by including only $\langle D 3 V\rangle$. The phonon frequencies $\left(\Omega_{\mu \mathbf{q}}\right)$ are obtained using Eq. (12) in the static limit, namely by using $\Pi_{\mu}(\mathbf{q}, 0)$.

we used a converged $12 \times 12 \times 12$ k-point grid in our $\mathrm{SnTe}$ calculations.

\section{APPENDIX C: EVALUATION OF PbTe THERMAL EXPANSION AT $600 \mathrm{~K}$}

It has been shown that the quasiharmonic approximation fails in describing the behavior of the transverse optical mode as a function of temperature [20]. In particular, the quasiharmonic approximation leads to strongly decreasing phonon frequencies in SnTe. Here we show that this error can be corrected by computing the thermal expansion in the framework of the self-consistent harmonic approximation and the calculating anharmonic phonon spectra.

To evaluate the effects of thermal expansion in our calculations for $\mathrm{PbTe}$ at $600 \mathrm{~K}$, we perform several SSCHA runs for different volumes using a $2 \times 2 \times 2$ supercell. We add the vibrational free energy to the $\mathrm{BO}$ total energy to construct an energy vs lattice parameter curve. By finding the minimum of this curve we obtain a lattice parameter of $6.642 \AA$ for PbTe at $600 \mathrm{~K}$ using the PBE functional in our calculations. We also estimate the thermal expansion coefficient $\gamma$. In PBE we find $\gamma=2.3 \times 10^{-5} \mathrm{~K}^{-1}$, in good agreement with the experimental value $\gamma=2.04 \times 10^{-5} \mathrm{~K}^{-1}$ obtained in Ref. [39]. With this new lattice constant, we then compute the anharmonic phonon dispersion as before. Figure 11 shows the phonon spectra for the two lattice parameters (PBE $T=0 \mathrm{~K}$ and $T=600 \mathrm{~K}$ ) considering just $\langle D 3 V\rangle$ and the case $\langle D 3 V\rangle+\langle D 4 V\rangle$. The phonon frequencies shift toward smaller values in relation to the PBE at $0 \mathrm{~K}$ as illustrated in Fig. 8. The shift is not significant for the $\langle D 3 V\rangle+\langle D 4 V\rangle$ case, whereas for the other, terms beyond the bubble gain more importance.

\section{APPENDIX D: BORN EFFECTIVE CHARGES AND DOPING}

The fact that both undoped $\mathrm{PbTe}$ and $\mathrm{SnTe}$ do not exist stoichiometric raises questions on how those systems should be simulated. For $\mathrm{PbTe}$, since the typical doping is quite small and the LO/TO splitting is quite pronounced, the inclusion of the Born effective charges is a natural choice. The effective charges for $\mathrm{PbTe}$ are calculated via DFPT and a posteriori added to the SSCHA dynamical matrices. On the other hand, on SnTe the effects of doping on the phonon dispersion are way more significant. Undoped SnTe should be ferroelectric, however, its nature depends on the carriers concentration. Typically $\mathrm{SnTe}$ is heavily hole doped [19]. As hole concentration increases, the transition temperature decreases down to a point in which the system remains cubic, even at low temperatures, hence losing the ferroelectric phase. In order to tackle this problem,
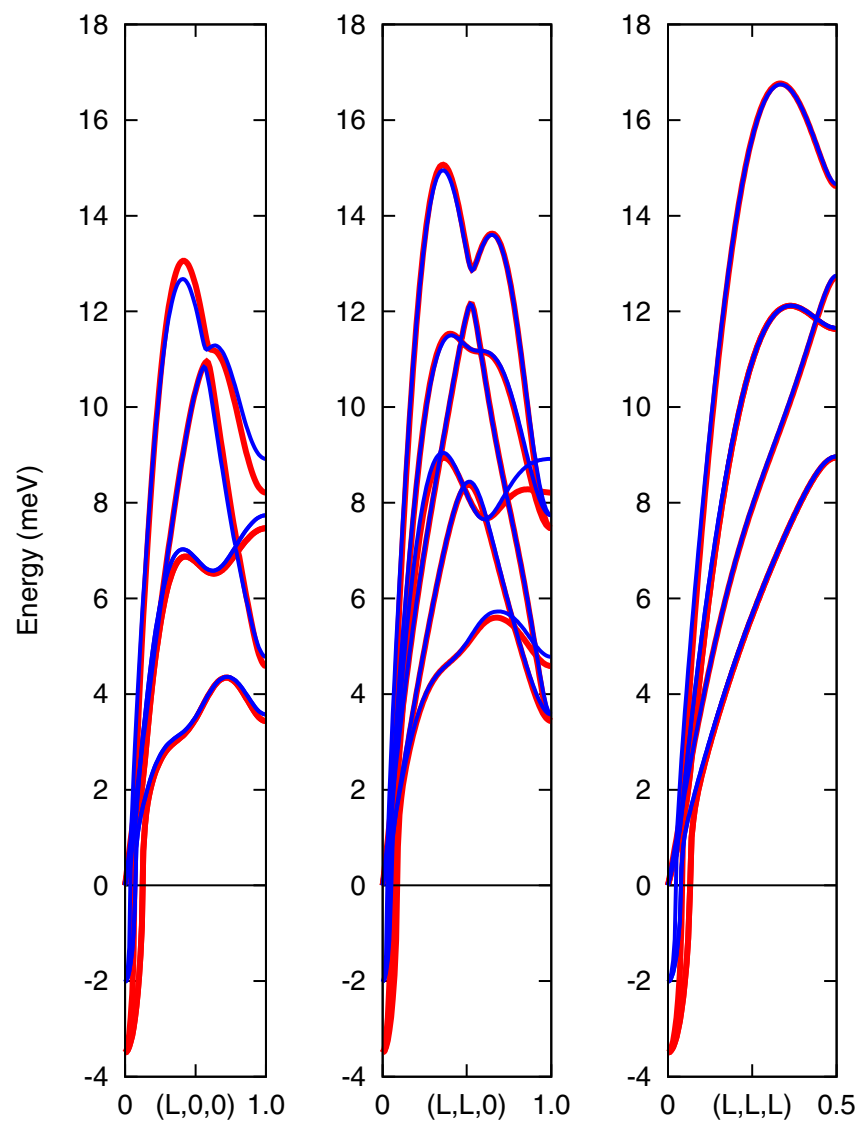

FIG. 12. SnTe harmonic phonon dispersion using a $4 \times 4 \times 4$ supercell: undoped (red) and doped (blue) case. Besides points very close to the zone center, doping does not change considerably the dispersion curves. 
we compared the harmonic phonon dispersion for doped and undoped SnTe on a $4 \times 4 \times 4$ supercell. We have not included the Born effective charges as they would be screened by doping, and we used $n_{h}=3.23 \times 10^{20} \mathrm{~cm}^{-3}$ from Ref. [18] as the carrier concentration. Figure 12 shows that the instability remains at this doping level, and the phonon dispersion along high symmetry directions are almost unaffected within the BZ.

\section{APPENDIX E: EMPIRICAL POTENTIAL CALCULATIONS}

In this paper, in order to investigate supercell size effects on the phonons modes, especially at the zone center, in addition to the full $a b$ initio calculations presented in the main text, we made use of a model potential based on the formulation developed by Marianetti et al. [40,41]. The potential has the form:

$$
V(\boldsymbol{R})=\frac{1}{2} \sum_{a b} \phi_{a b} u^{a} u^{b}+V_{A}^{(3)}(\boldsymbol{u})+V_{A}^{(4)}(\boldsymbol{u}),
$$

where $\boldsymbol{u}=\boldsymbol{R}-\boldsymbol{R}_{(0)}, \boldsymbol{R}_{(0)}$ corresponding to the equilibrium configuration on the rock-salt structure. The harmonic matrices $\phi_{a b}$ were calculated using the same parameters as for the DFPT calculations described on the main text. Anharmonic terms $V_{A}^{(3)}$ and $V_{A}^{(4)}$ are defined as:

$$
V_{A}^{(3)}(\boldsymbol{u})=p_{3} \sum_{s=1}^{N_{a}} \sum_{\alpha=x, y, z}\left[\mathcal{A}_{s, \alpha_{+}}^{3}-\mathcal{A}_{s, \alpha_{-}}^{3}\right]
$$

and

$$
\begin{aligned}
V_{A}^{(4)}(\boldsymbol{u})= & p_{4} \sum_{s=1}^{N_{a}} \sum_{\alpha=x, y, z}\left[\mathcal{A}_{s, \alpha_{+}}^{4}+\mathcal{A}_{s, \alpha_{-}}^{4}\right] \\
& +p_{4 x} \sum_{s=1}^{N_{a}} \sum_{\alpha=x, y, z}\left[\mathcal{A}_{s, \alpha_{+}}^{2}\left(\left(\mathcal{E}_{s, \alpha_{+}}^{(1)}\right)^{2}+\left(\mathcal{E}_{s, \alpha_{+}}^{(2)}\right)^{2}\right)\right. \\
& \left.+\mathcal{A}_{s, \alpha_{-}}^{2}\left(\left(\mathcal{E}_{s, \alpha_{-}}^{(1)}\right)^{2}+\left(\mathcal{E}_{s, \alpha_{-}}^{(2)}\right)^{2}\right)\right],
\end{aligned}
$$

where, for example

$$
\begin{aligned}
& \mathcal{A}_{s, x_{ \pm}}=\frac{1}{\sqrt{2}}\left(u^{x_{ \pm}(s), x}-u^{s, x}\right) \\
& \mathcal{E}_{s, x_{ \pm}}^{(1)}=\frac{1}{\sqrt{2}}\left(u^{x_{ \pm}(s), y}-u^{s, y}\right) \\
& \mathcal{E}_{s, x_{ \pm}}^{(2)}=\frac{1}{\sqrt{2}}\left(u^{x_{ \pm}(s), z}-u^{s, z}\right) .
\end{aligned}
$$

The variables $x_{+}(s)$ and $x_{-}(s)$ represent the nearest neighbor of atom $s$, along the Cartesian direction $+x$ and $-x$, respectively. For the other Cartesian directions, $\pm y$ and $\pm z$, we generalized this notation. The quantity $u$ is the displacement from the equilibrium position.

The potentials for both systems were defined by fitting the parameters $p_{3}, p_{4}$, and $p_{4 x}$ to ab initio forces calculated for 1000 random atomic configurations generated in the firstprinciples SSCHA calculation. For PbTe we used a combination of configurations generated at $300 \mathrm{~K}$ and $600 \mathrm{~K}$, resulting on the coefficients $p_{3}=2.99 \mathrm{eV} /(\AA)^{3}, p_{4}=4.17 \mathrm{eV} /(\AA)^{4}$, and $p_{4 x}=-1.32 \mathrm{eV} /(\AA)^{4}$. For SnTe we used configurations

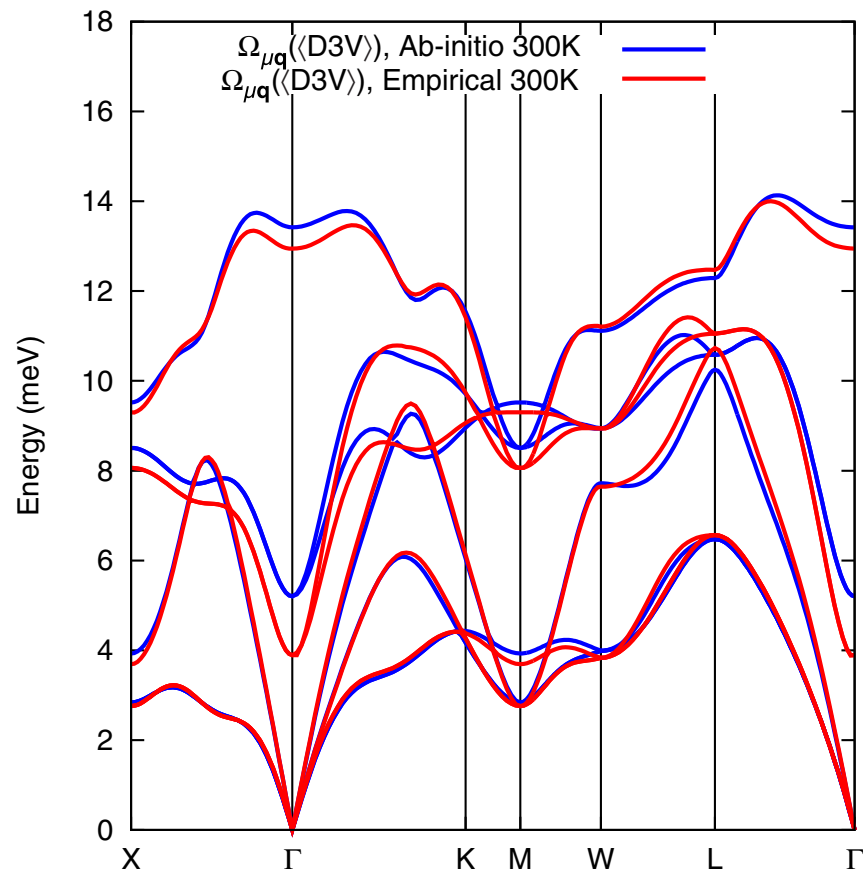

FIG. 13. Anharmonic PbTe phonon dispersion at $300 \mathrm{~K}$ on a $4 \times 4 \times 4$ supercell: $a b$ initio vs (blue lines) empirical potential (red lines).

generated at $100 \mathrm{~K}$ obtaining $p_{3}=2.51 \mathrm{eV} /(\AA)^{3}$ and $p_{4}=$ $6.18 \mathrm{eV} /(\AA)^{4}$, in this case $p_{4 x}$ was neglected since its contribution was not relevant.

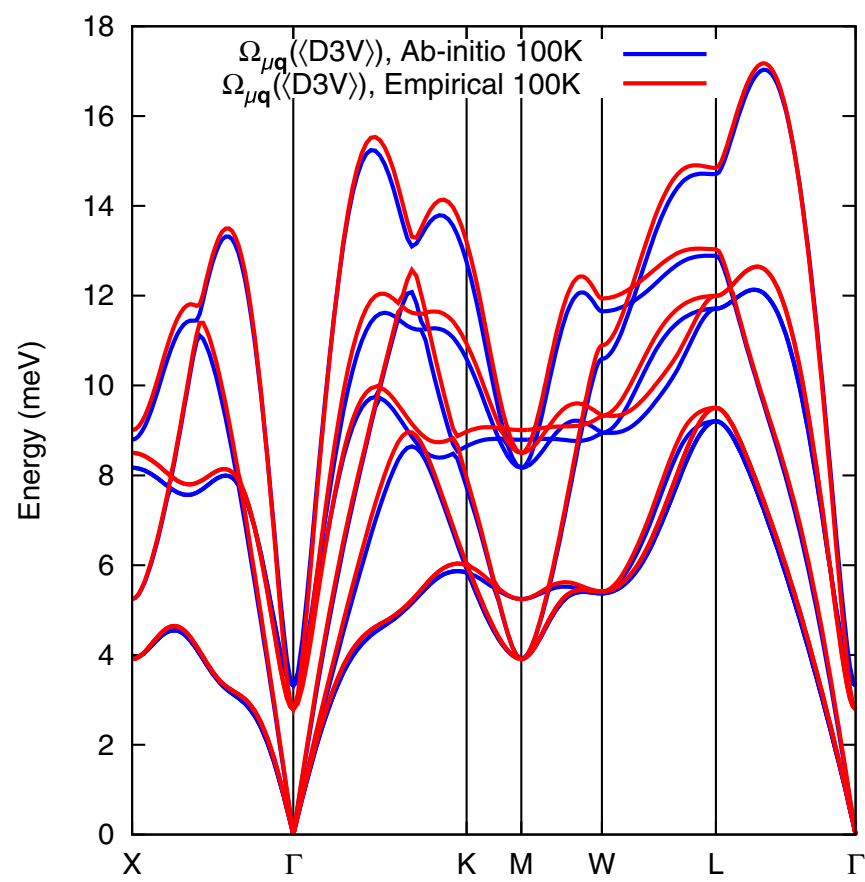

FIG. 14. Anharmonic SnTe phonon dispersion at $100 \mathrm{~K}$ on a $4 \times 4 \times 4$ supercell: $a b$ initio vs (blue lines) empirical potential (red lines). The phonon frequencies $\left(\Omega_{\mu \mathbf{q}}\right)$ are obtained using Eq. (12) in the static limit, namely by using $\Pi_{\mu}(\mathbf{q}, 0)$. 


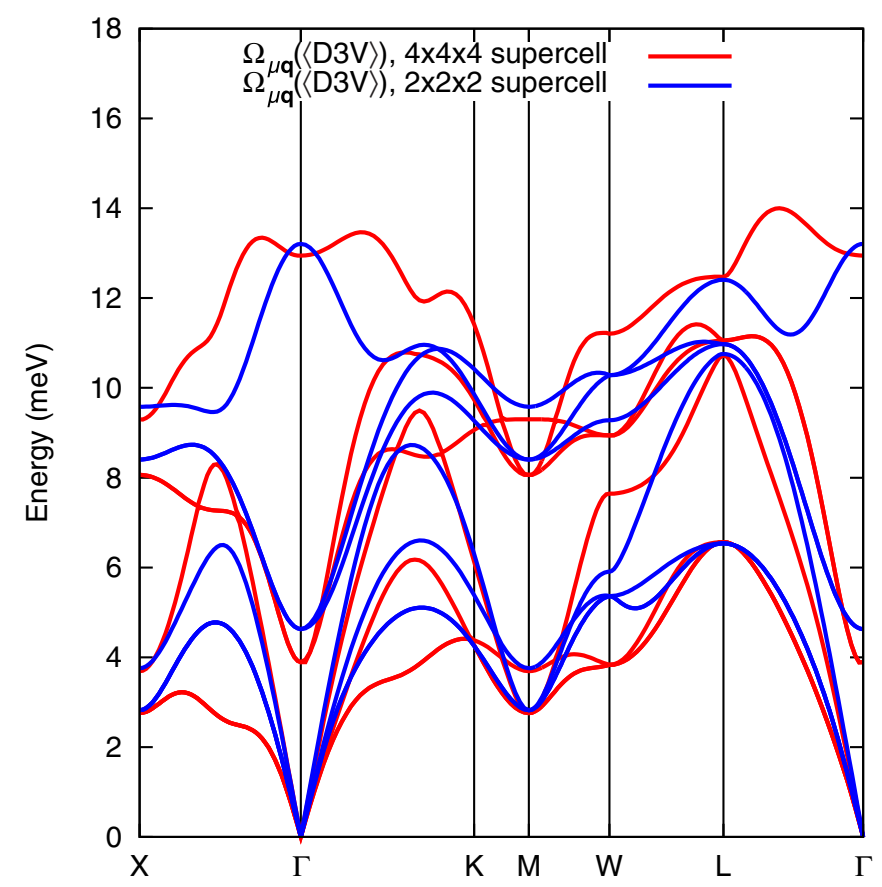

FIG. 15. Comparison between PbTe anharmonic phonon spectra using a $2 \times 2 \times 2$ (blue) and $4 \times 4 \times 4$ (red) supercell. Both dispersions were calculated using an empirical potential and including the bubble term. The phonon frequencies $\left(\Omega_{\mu \mathbf{q}}\right)$ are obtained using Eq. (12) in the static limit, namely by using $\Pi_{\mu}(\mathbf{q}, 0)$.

Figures 13 and 14 compare the phonon dispersion on a $4 \times 4 \times 4$ supercell calculated $a b$ initio and using the empirical potential for PbTe $(300 \mathrm{~K})$ and $\mathrm{SnTe}(100 \mathrm{~K})$, respectively. For

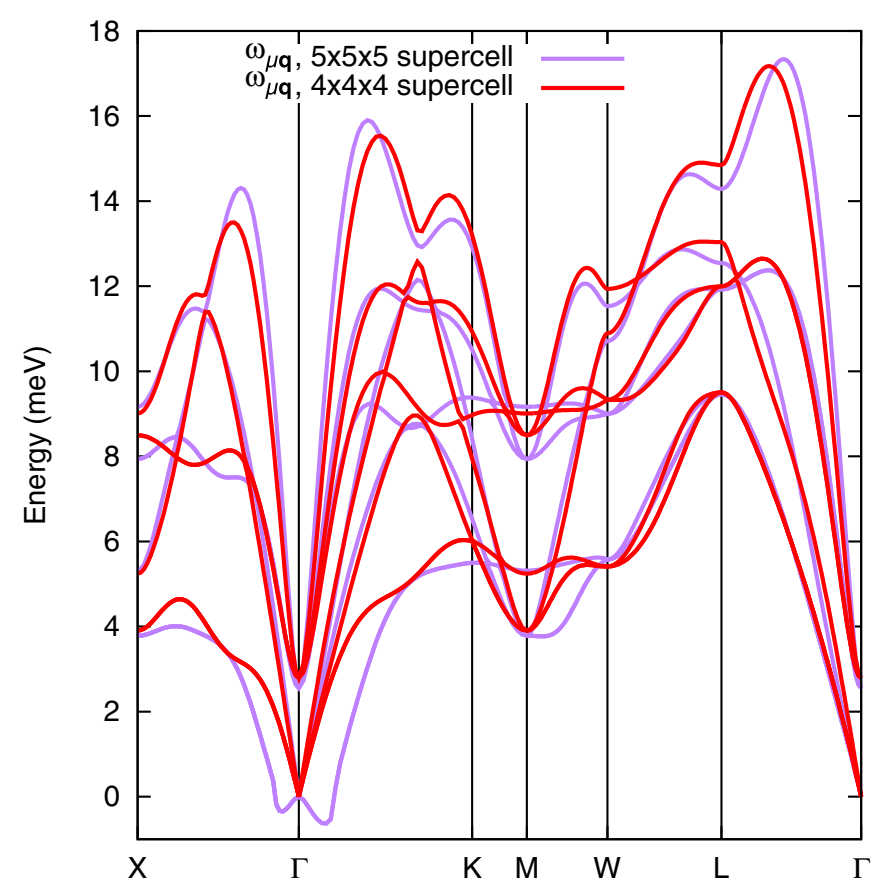

FIG. 16. Comparison between SnTe anharmonic phonon spectra using a $4 \times 4 \times 4$ (red) and $5 \times 5 \times 5$ (purple) supercell. In this case the bubble and higher order terms are neglected on our calculations. The phonon frequencies $\left(\omega_{\mu \mathbf{q}}\right)$ are obtained using Eq. (12).
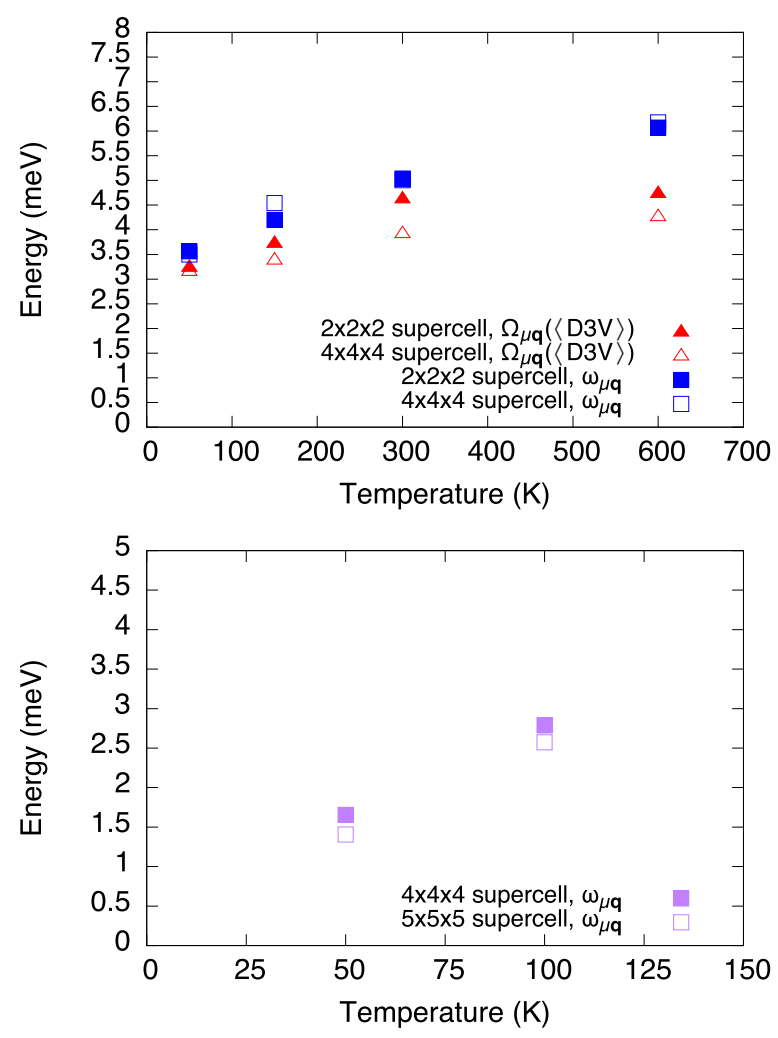

FIG. 17. Transverse optical phonon modes as a function of $T$ for PbTe (top) and SnTe (bottom) for different supercell sizes. The bubble contribution was taken on for supercells up to the $4 \times 4 \times 4$. The phonon frequencies $\left(\Omega_{\mu \mathbf{q}}\right)$ are obtained using Eq. (12) in the static limit, namely by using $\Pi_{\mu}(\mathbf{q}, 0)$.

$\mathrm{PbTe}$ the larger difference is at zone center, this may be due to the fact that we used random configurations generated on a broader range of temperatures. However, this is not a problem in order to study the convergence of the TO modes using the empirical potential for different supercell sizes. For SnTe, since we generated our random configurations at $100 \mathrm{~K}$, one may expect a better agreement.

Figure 17 presents the convergence tests regarding the TO modes of $\mathrm{PbTe}$ and $\mathrm{SnTe}$. For the first compound, the difference between the $2 \times 2 \times 2$ and $4 \times 4 \times 4$ is small for the points which are included exactly by using the $2 \times 2 \times 2$ supercell $(X, \Gamma, L)$, as shown in Fig. 15. However, as stated before, we used the $4 \times 4 \times 4$ supercell in order to include more points along on the $\mathrm{BZ}$ and, as a consequence, to describe more accurately the phonon dispersion of PbTe. As mentioned on the main text, for SnTe we considered at least a $4 \times 4 \times 4$ supercell in our ab initio calculations, since the $2 \times 2 \times 2$ does not seem to be sufficient. In order to test the convergence we explored the model potential on the $4 \times 4 \times 4$ and also on the $5 \times 5 \times 5$ supercells. In this case, we compared just the SSCHA runs without including extra terms. Figure 16 shows the phonon dispersion for the $4 \times 4 \times 4$ and $5 \times 5 \times 5$ supercell. It is necessary to emphasize that the latter presents some wiggles due to the Fourier transform, so the negative energies are not physical, just an interpolation artifact. As can be viewed in Fig. 17, the difference between the TO modes using different supercells is not significant for our purposes. 
[1] H. J. Goldsmid, Introduction to Thermoelectricity (Springer, Berlin, 2010).

[2] K. Behnia, Fundamentals of Thermoelectricity (Oxford University Press, Oxford, 2015).

[3] X. Zheng, C. Liu, Y. Yan, and Q. Wang, Renew. Sust. Energ. Rev. 32, 486 (2014).

[4] M. H. Elsheikh, D. A. Shnawah, M. F. M. Sabri, S. B. M. Said, M. H. Hassan, M. B. A. Bashir, and M. Mohamad, Renew. Sust. Energ. Rev. 30, 337 (2014).

[5] G. Chen, M. S. Dresselhaus, G. Dresselhaus, J.-P. Fleurial, and T. Caillat, Int. Mater. Rev. 48, 45 (2003).

[6] A. van Roekeghem, J. Carrete, and N. Mingo, Phys. Rev. B 94, 020303 (2016).

[7] O. Hellman, P. Steneteg, I. A. Abrikosov, and S. I. Simak, Phys. Rev. B 87, 104111 (2013).

[8] P. Souvatzis, O. Eriksson, M. I. Katsnelson, and S. P. Rudin, Phys. Rev. Lett. 100, 095901 (2008).

[9] I. Errea, M. Calandra, and F. Mauri, Phys. Rev. Lett. 111, 177002 (2013)

[10] I. Errea, M. Calandra, and F. Mauri, Phys. Rev. B 89, 064302 (2014).

[11] G. Lang, K. Karch, M. Schmitt, P. Pavone, A. P. Mayer, R. K. Wehner, and D. Strauch, Phys. Rev. B 59, 6182 (1999).

[12] M. Lazzeri and S. de Gironcoli, Phys. Rev. B 65, 245402 (2002).

[13] G. S. Pawley, W. Cochran, R. A. Cowley, and G. Dolling, Phys. Rev. Lett. 17, 753 (1966).

[14] R. A. Cowley, Rep. Prog. Phys. 31, 123 (1968).

[15] W. Cochran, R. A. Cowley, G. Dolling, and M. Elcombe, Proc. R. Soc. Lond. A 293, 433 (1966).

[16] O. Delaire, J. Ma, K. Marty, A. May, M. McGuire, M.-H. Du, D. Singh, A. Podlesnyak, G. Ehlers, M. Lumsden, and B. Sales, Nat. Mater. 10, 614 (2011).

[17] C. W. Li, J. Ma, H. B. Cao, A. F. May, D. L. Abernathy, G. Ehlers, C. Hoffmann, X. Wang, T. Hong, A. Huq, O. Gourdon, and O. Delaire, Phys. Rev. B 90, 214303 (2014).

[18] C. D. O'Neill, D. A. Sokolov, A. Hermann, A. Bossak, C. Stock, and A. D. Huxley, Phys. Rev. B 95, 144101 (2017).

[19] K. L. I. Kobayashi, Y. Kato, Y. Katayama, and K. F. Komatsubara, Phys. Rev. Lett. 37, 772 (1976).

[20] A. H. Romero, E. K. U. Gross, M. J. Verstraete, and O. Hellman, Phys. Rev. B 91, 214310 (2015).

[21] C. W. Li, O. Hellman, J. Ma, A. F. May, H. B. Cao, X. Chen, A. D. Christianson, G. Ehlers, D. J. Singh, B. C. Sales, and O. Delaire, Phys. Rev. Lett. 112, 175501 (2014).
[22] K. M. Rabe and J. D. Joannopoulos, Phys. Rev. B 32, 2302 (1985).

[23] T. Shiga, J. Shiomi, J. Ma, O. Delaire, T. Radzynski, A. Lusakowski, K. Esfarjani, and G. Chen, Phys. Rev. B 85, 155203 (2012).

[24] J. An, A. Subedi, and D. Singh, Solid State Commun. 148, 417 (2008).

[25] D. Hooton, Philos. Mag. 46, 422 (1955).

[26] E. R. Cowley, J. K. Darby, and G. S. Pawley, J. Phys. C 2, 1916 (1969).

[27] R. Bianco, I. Errea, L. Paulatto, M. Calandra, and F. Mauri, Phys. Rev. B 96, 014111 (2017).

[28] P. Giannozzi, S. Baroni, N. Bonini, M. Calandra, R. Car, C. Cavazzoni, D. Ceresoli, G. L. Chiarotti, M. Cococcioni, I. Dabo, A. D. Corso, S. de Gironcoli, S. Fabris, G. Fratesi, R. Gebauer, U. Gerstmann, C. Gougoussis, A. Kokalj, M. Lazzeri, L. Martin-Samos, N. Marzari, F. Mauri, R. Mazzarello, S. Paolini, A. Pasquarello, L. Paulatto, C. Sbraccia, S. Scandolo, G. Sclauzero, A. P. Seitsonen, A. Smogunov, P. Umari, and R. M. Wentzcovitch, J. Phys.: Condens. Matter 21, 395502 (2009).

[29] J. P. Perdew, K. Burke, and M. Ernzerhof, Phys. Rev. Lett. 77, 3865 (1996).

[30] N. Troullier and J. L. Martins, Phys. Rev. B 43, 1993 (1991).

[31] P. E. Blöchl, Phys. Rev. B 50, 17953 (1994).

[32] S. Baroni, S. de Gironcoli, A. Dal Corso, and P. Giannozzi, Rev. Mod. Phys. 73, 515 (2001).

[33] R. Dalven, Infrared Phys. 9, 141 (1969).

[34] G. N. Schlicht, Narrow-Gap Lead Salts (Springer, Berlin, 2006).

[35] W. Jantsch, Springer Tracts Mod. Phys. 99, 486 (1983).

[36] A. J. Miller, G. A. Saunders, and Y. K. Yogurtcu, J. Phys. C: Solid State Phys. 14, 1569 (1981).

[37] S. S. Kabalkina, N. R. Serebryanaya, and L. F. Vereshchagin, Fiz. Tverd. Tela (Leningrad) 10, 733 (1968) [Sov. Phys. Solid State 10, 574 (1968)].

[38] D. Bansal, J. Hong, C. W. Li, A. F. May, W. Porter, M. Y. $\mathrm{Hu}$, D. L. Abernathy, and O. Delaire, Phys. Rev. B 94, 054307 (2016).

[39] B. Houston, R. E. Strakna, and H. S. Belson, J. Appl. Phys. 39, 3913 (1968).

[40] X. Ai, Y. Chen, and C. A. Marianetti, Phys. Rev. B 90, 014308 (2014).

[41] Y. Chen, X. Ai, and C. A. Marianetti, Phys. Rev. Lett. 113, 105501 (2014). 